\title{
INDEPENDENT TRANSVERSAL TOTAL DOMINATION VERSUS TOTAL DOMINATION IN TREES
}

\author{
Abel Cabrera Martínez \\ Departament d'Enginyeria Informàtica i Matemàtiques \\ Universitat Rovira i Virgili \\ Av. Països Catalans 26, 43007 Tarragona, Spain \\ e-mail: abel.cabrera@urv.cat \\ IZTOK PETERIN \\ Faculty of Electrical Engineering and Computer Science \\ University of Maribor \\ Koroška cesta 46, 2000 Maribor, Slovenia \\ e-mail: iztok.peterin@um.si \\ AND \\ ISMAEL G. Yero \\ Departamento de Matemáticas \\ Escuela Politécnica Superior de Algeciras, Universidad de Cádiz \\ Av. Ramón Puyol s/n, 11202 Algeciras, Spain \\ e-mail: ismael.gonzalez@uca.es
}

\begin{abstract}
A subset of vertices in a graph $G$ is a total dominating set if every vertex in $G$ is adjacent to at least one vertex in this subset. The total domination number of $G$ is the minimum cardinality of any total dominating set in $G$ and is denoted by $\gamma_{t}(G)$. A total dominating set of $G$ having nonempty intersection with all the independent sets of maximum cardinality in $G$ is an independent transversal total dominating set. The minimum cardinality of any independent transversal total dominating set is denoted by $\gamma_{t t}(G)$. Based on the fact that for any tree $T, \gamma_{t}(T) \leq \gamma_{t t}(T) \leq \gamma_{t}(T)+1$, in this work we give several relationships between $\gamma_{t t}(T)$ and $\gamma_{t}(T)$ for trees $T$ which are leading to classify the trees which are satisfying the equality in these bounds.
\end{abstract}

Keywords: independent transversal total domination number, total domination number, independence number, trees.

2010 Mathematics Subject Classification: 05C69, 05 C05. 


\section{REFERENCES}

[1] C. Brause, M.A. Henning and M. Krzywkowski, A characterization of trees with equal 2-domination and 2-independence numbers, Discrete Math. Theor. Comput. Sci. 19 (2017) \#1.

doi:10.23638/DMTCS-19-1-1

[2] A. Cabrera Martínez, J.M. Sigarreta Almira and I.G. Yero, On the independence transversal total domination number of graphs, Discrete Appl. Math. 219 (2017) 65-73.

doi:10.1016/j.dam.2016.10.033

[3] M. Chellali and T.W. Haynes, A note on the total domination of a tree, J. Combin. Math. Combin. Comput. 58 (2006) 189-193.

[4] M.A. Henning, A survey of selected recent results on total domination in graphs, Discrete Math. 309 (2009) 32-63.

doi:10.1016/j.disc.2007.12.044

[5] M.A. Henning and S.A. Marcon, A constructive characterization of trees with equal total domination and disjunctive domination numbers, Quaest. Math. 39 (2016) 531-543.

doi:10.2989/16073606.2015.1096860

[6] M.A. Henning and A. Yeo, Total Domination in Graphs (Springer, New York, 2013). doi:10.1007/978-1-4614-6525-6

[7] Z. Li and J. Xu, A characterization of trees with equal independent domination and secure domination numbers, Inform. Process. Lett. 119 (2017) 14-18.

doi:10.1016/j.ipl.2016.11.004

Received 6 June 2018

Revised 11 September 2018

Accepted 12 September 2018 